TITLE:

\title{
Three-dimensional turbulence structure of rectangular side-cavity zone in open-channel streams
}

\author{
$\operatorname{AUTHOR}(\mathrm{S})$ : \\ Sanjou, Michio; Akimoto, Tetsuro; Okamoto, \\ Takaaki
}

\section{CITATION:}

Sanjou, Michio ... [et al]. Three-dimensional turbulence structure of rectangular side-cavity zone in open-channel streams. International Journal of River Basin Management 2012, 10(4): 293-305

ISSUE DATE:

2012-09-10

URL:

http://hdl.handle.net/2433/178725

\section{RIGHT:}

(C) 2012 International Association for Hydro-Environment Engineering and Research; This is not the published version. Please cite only the published version.; この論文は出版社版でありません。引用の際には 出版社版をご確認ご利用ください。 


\title{
THREE-DIMENSIONAL TURBULENCE STRUCTURE OF RECTANGULAR SIDE-CAVITY
}

\section{ZONE IN OPEN-CHANNEL STREAM}

\author{
By Michio Sanjou $^{1}$, Tetsuro Akimoto ${ }^{2}$ and Takaaki Okamoto ${ }^{3}$
}

\begin{abstract}
Open-ended side-cavity zones are often observed in natural rivers. They appear in embayment and aligned spur-dike fields. Many pollutant clouds and suspended sediments are conveyed and trapped in the cavities, and it is thus very important in environmental hydraulics and river management to accurately predict mass and momentum exchanges through mainstream/side-cavity interface. It is well known that small-scale shedding vortices are generated due to shear instability induced by velocity differences between high-speed mainstream and low-speed embayment flows. A large momentum in the main channel causes large-scale horizontal gyre in the cavity zone. The coherent turbulence structure in the mainstream/embayment boundary and the horizontal large-scale gyre structure play a key role in the mass/momentum exchanges. In particular, previous studies have pointed out that a rectangular-shaped cavity zone with an aspect ratio of 3.0 produces two kinds of gyres, and shows a more effective exchange property in comparison with a square-shaped cavity. However, much uncertainty remains regarding the detailed hydrodynamics accompanied by three-dimensional turbulence motions.

Using large eddy simulation, which is also compared with particle image velocimetry measurements, we predict three-dimensional current properties and turbulence structure. Based on these results, a significant relation between instantaneous vertical flows and the spanwise momentum transfer is shown. Furthermore, a phenomenological flow model is proposed for the side-cavity zone in the open-channel field.
\end{abstract}

Keywords: open-channel flow, side-cavity zone, numerical simulation and mass/momentum exchanges

\footnotetext{
${ }^{1}$ Associate Professor, Department of Civil Engineering, Kyoto University, Kyoto 615-8540, Japan ${ }_{2}^{2}$ Graduate school student, Department of Civil Engineering, Kyoto University, Kyoto 615-8540, Japan

${ }^{3}$ Assistant Professor, Department of Civil Engineering, Kyoto University, Kyoto 615-8540, Japan
} 


\section{INTRODUCTION}

There are many dead-water zones composed of consecutive groynes in natural streams. The groynes and spur dikes are generally constructed in banks to prevent bank erosion and to control the flow direction. Dead-water zones can also be found in natural harbors in rivers. A velocity gap is very remarkable between the mainstream and the embayment, and thus, flow patterns become very unstable. This phenomenon is called shear instability. Coherent vortices, such as the Karman vortex, are then generated periodically in the mainstream/embayment interface region forming a typical mixing layer, as pointed out by Booij (2004). Furthermore, the mainstream momentum induces a large-scale horizontal circulation in the side cavity. Such a large gyre may catch a pollutant cloud and suspended sediments locally. It is therefore of vital importance in river management and ecohydraulics to understand the generation mechanisms of these circulations and the correlated mass/momentum transfers through the junction between the main channel and the cavity zone.

Many researchers have tried to these exchange properties from various points of view. For example, Yossef and Vriend (2010) studied sedimentation properties around the groyne; they conducted laboratory measurements in a mobile-bed flume and compared several hydrodynamic values, such as velocity, free-surface fluctuation, and bed profiles, for various hydraulic conditions. Uijttewaal et al. (2001) conducted dye experiments and found a relationship between the aspect ratio, which is a ratio of longitudinal length to width of side cavity, and the exchange rate through the mainstream/embayment boundary. Their results suggested that a mass transfer coefficient depends on the geometry of groynes, water depth, groyne rank number, and bed shape. Of particular significance is that the aspect ratio of the rectangular embayment is a key factor in defining the mass transfer coefficient. In a square embayment, in which the aspect ratio is 1.0, a single horizontal gyre is observed, and the mass transfer is dominated by the mixing layer formed along the boundary between the main stream and the cavity zone. Generally, the dye remains in the center region of the gyre, because the mixing layer is not penetrated to within the horizontal gyre. On the other hand, in a large groyne field, i.e., one with a large aspect ratio, coherent shedding vortices are produced from a tip of the upper lank groyne. These structures are conveyed to the center of the primary gyre, and this causes the effective mass exchange compared with the square-shaped embayment field. It should be noted that in a mass such as dye, pollutant sediments are trapped in the secondary gyre, which is shielded from the primary gyre, shedding vortices, and the mixing layer. 
Weitbrecht et al. (2008) conducted laboratory measurements and obtained velocity distributions and dye concentration data. They studied the relation between the exchange rate and the bed configuration of the cavity, and determined the mass transfer coefficient by using velocity components and the time-decay of concentration within the embayment. They pointed out that the mass transfer coefficient has a linear relation with the normalized morphometric groyne field parameter, which consists of water depth, and width and length of groyne.

Many researchers have treated these canopy fields as two-dimensional shallow flows. Therefore, not much is known about the three-dimensional hydrodynamic characteristics. It is generally difficult to approach these topics by measurement methods. Thus, some research groups introduce numerical simulations, such as Reynolds-averaged Navier-Stokes solver, large eddy simulation (LES), and detached eddy simulation (e.g., Chang et al. 2006; Chang et al. 2007; Hinterberger et al. 2007; Abad et al. 2008; McCoy et al. 2008). Constantinescu et al. (2009) developed a three-dimensional LES and simulated dissolved contaminant exchange through the main-channel/embayment interface. Their numerical results suggest that the ejection and exchange processes are very non-uniform over the depth. Many contaminants are swept away from the dead-water zone. Such three-dimensionality of mass transfer was very important in evaluating the mass/momentum exchanges and turbulence transport in the embayment. Unfortunately, much uncertainty remains about the three-dimensional turbulence structure within and around the open-cavity area in natural rivers.

Thus, the present paper introduces the three-dimensional LES and describes the turbulence structure and flow patterns in the embayment in detail. Particle image velocimetry (PIV) measurements are also conducted to examine the accuracy of the present numerical model. Furthermore, we quantitatively predict the momentum transfer properties through the mainstream/cavity interface and the related instantaneous flow fields. Section 2 describes the numerical method, basic equations, experiment procedure, and hydraulic conditions. Section 3 discusses the mean velocity fields using LES and PIV data. In particular, we focus on the flow patterns in three kinds of planes with different directions, i.e., horizontal, vertical, and cross-sectional planes. Furthermore, we examine the calculation accuracy of the present LES by comparison with the PIV results. In section 4, we describe instantaneous velocity fields with a strong three-dimensional structure. With the introduction of a conditional sampling technique, spanwise momentum transfer can be evaluated reasonably. In section 5, we propose a 
phenomenological flow model for time-averaged horizontal gyres and instantaneous momentum transfer in and around the side-cavity zone in the open-channel flow. Section 6 presents the conclusions.

\section{CALCULATION AND MEASUREMENT METHODS}

\subsection{Flow field and hydraulic condition}

Figure 1 shows the flow field and coordinate system in the present study, where $H$ is the water depth, $B$ is the main-channel width, and $B_{w}$ and $L_{w}$ are width and streamwise length of the side cavity, respectively. The ratio of $L_{w} / B_{w}$, generally called the aspect ratio, is chosen to be 3.0 . This aspect ratio is often observed in natural embayment and groyne fields in Japanese rivers. It is also well known that a side-cavity zone with an aspect ratio of 3.0 has quite different hydrodynamic characteristics from that of a square-shaped cavity with an aspect ratio of 1.0, and mass/momentum exchanges are promoted significantly by the primary and secondary horizontal gyres. The bulk-mean velocity of the main-channel $U_{m}$ is $12 \mathrm{~cm} / \mathrm{s}, H=5 \mathrm{~cm}, B=30 \mathrm{~cm}, B_{w}=10 \mathrm{~cm}$, and $L_{w}$ $=30 \mathrm{~cm}$

The streamwise, vertical, and spanwise directions are denoted by $x, y$, and $z$, respectively, whereas $\tilde{u}, \widetilde{v}$ and $\widetilde{w}$ are the instantaneous velocity components for the corresponding directions. They could be decomposed into mean components and their fluctuations in the following ways:

$$
\begin{aligned}
& \tilde{u}=U+u \\
& \widetilde{v}=V+v \\
& \widetilde{w}=W+w
\end{aligned}
$$

where the large and the small characters indicate the mean and the turbulent components, respectively. The origins of $y$ and $z$ are the flume bottom and sidewall of the cavity, and thus, the main-channel/embayment interface corresponds with $z / B_{w}=1.0$. The cavity zone and the mainstream are situated in $z / B_{w}<1.0$ and $z / B_{w}>1.0$, respectively.

\subsection{Calculation method}

The LES is a powerful calculation technique, because it can predict the existence of large-scale coherent eddies very well. Since Moin and Kim (1982) successfully calculated bursting 
phenomena reasonably, many researchers have applied the technique intensively to numerically predict three-dimensional turbulence flows. Authors have also conducted numerical studies on compound open-channel flows and vegetated free-surface streams, and reproduced turbulence transports using the LES (see Sanjou \& Nezu 2011 and Okamoto \& Nezu 2010).

Generally, the LES solves filtered momentum equations. The filtered continuity equation and N-S equation are described as follows:

$$
\begin{gathered}
\frac{\partial\left\langle\tilde{u}_{i}\right\rangle}{\partial x_{i}}=0 \quad(i=1,2,3) \\
\frac{\partial\left\langle\tilde{u}_{i}\right\rangle}{\partial t}+\left\langle\tilde{u}_{j}\right\rangle \frac{\partial\left\langle\tilde{u}_{i}\right\rangle}{\partial x_{j}}=F_{i}-\frac{\partial\langle\tilde{p}\rangle}{\partial x_{i}}+\frac{\partial}{\partial x_{i}}\left(-\tau_{i j}+2 v\left\langle\widetilde{D}_{i j}\right\rangle\right) \\
F_{i}=(g \sin \theta,-g \cos \theta, 0) \\
D_{i j} \equiv \frac{1}{2}\left(\frac{\partial \tilde{u}_{i}}{\partial x_{j}}+\frac{\partial \tilde{u}_{j}}{\partial x_{i}}\right)
\end{gathered}
$$

where the bracket $<>$ denotes the filtering operation in space, and $\tau_{i j}$ is the sub-grid scale (SGS) stress tensor calculated by the standard Smagorinsky model, as follows:

$$
\tau_{i j}=-2 v_{T}\left\langle D_{i j}\right\rangle
$$

When the SGS variances are assumed to be isotropic, the SGS eddy viscosity $v_{T}$ is given by

$$
v_{T}=\left(C_{s} \Delta\right)^{2}\left(2\left(D_{i j}\right)\left(D_{i j}\right\rangle\right)^{1 / 2} \text { and } \Delta=\left(\Delta_{1} \Delta_{2} \Delta_{3}\right)^{1 / 3}
$$

where $C_{s}$ is the Smagorinsky constant; $C_{s}=0.15$ was chosen in this study. $\Delta$ is the SGS filtering scale, which corresponds to the computational grid scale. The third-order upwind difference scheme was used for the convection term, and the second-order Adams-Bashforth method was adopted for the time differentiation. The computational points were displaced in the rectangular grid, and the number of the calculated grid was set at 190, 30, and 89 for the streamwise, vertical, and spanwise directions, respectively. The corresponding grid sizes were $5 \mathrm{~mm}, 2 \mathrm{~mm}$, and $5 \mathrm{~mm}$, except in the near wall regions and in the main-channel/embayment boundary where the finer sizes were chosen. 
In the present study, a finite-volume driver zone was placed in the upstream side of the cavity in the calculation region. We reproduced a fully developed turbulence flow in the driver zone under the cyclic boundary condition in the streamwise direction, and these developed flows were imposed on the inflow condition of the main-calculation zone. No-slip condition was given at all rigid walls. The free-surface boundary was calculated using the volume-of-fluid method proposed by Hirt and Nicholds (1981). The grid-scale velocity component $\left\langle\widetilde{u}_{i}\right\rangle$ means the instantaneous velocity component $\tilde{u}_{i}$ in order to avoid confusion in the following sections.

\subsection{PIV Measurements}

To examine calculation accuracy, a laboratory experiment was conducted for the same hydraulic condition as mentioned in section 2.1. PIV is a very useful fluid measurement method, because it can obtain time series of two-dimensional velocity components at multiple points. Thus, it allows us to reasonably understand coherent motions and turbulent eddies. Nezu and Sanjou (2011) introduced the various PIV results of open-channel turbulence.

The present experiments were conducted in a glass-made flume (length, $10 \mathrm{~m}$; width, $40 \mathrm{~cm}$ ), and a measured region was located at about $7 \mathrm{~m}$ downstream from the channel entrance. A continuous 2-W Ar-ion laser was used for a laser light sheet (LLS). The 2mm-thick LLS was projected along the centerline of the channel from the bottom. The spatial resolution was about $0.37 \mathrm{~mm}$ per pixel. Two kinds of experiments were conducted: one measuring the vertical plane, the other measuring the horizontal plane. The vertical $x-y$ plane was illuminated with tracer particles and was taken by a high-speed CMOS camera controlled by a personal computer connected with a pulse-signal generator. A trigger signal having the same frequency as data sampling rate $1 / \Delta t(\mathrm{~Hz})$ was transferred to the CMOS camera via the control computer, and a pair of digital images were taken accurately at the frame rate $1 / \Delta \delta(\mathrm{Hz})$ of the CMOS camera. In this study, $1 / \Delta t$ was set at 30 $\mathrm{Hz}$ and $1 / \Delta \delta$. was set at $100 \mathrm{~Hz}$. The total sampling time was 60 seconds. The horizontal $x-z$ plane experiment was also conducted in the same fashion. The time-variation of the instantaneous velocity vectors was calculated by a PIV algorithm, in which the interrogation size was $29 \times 29$ pixels. When a correlation value between the first and the second image patterns was less than 0.4, a local velocity vector was judged an invalid vector, and instead of it, an interpolated velocity data was given to the corresponding position by using surrounding valid vectors. We obtained spatial distributions of mean and turbulence velocity components. 


\section{MEAN VELOCITY FIELDS IN THE EMBAYMENT}

\subsection{Horizontal distribution of mean velocity vectors}

Figure 2 shows the distribution of the mean velocity components $(U, W)$ in the horizontal plane at $y$ $/ H=0.2,0.4$, and 0.8 , in which measured data by PIV are also indicated. We can see two gyre structures in the measured results. One large-scale horizontal gyre induced by the velocity shear between the main channel and the embayment is called the primary gyre (PG). The other gyre is produced by the PG and is called the secondary gyre (SG). Uijitewaal et al. (2001) and Uijitewaal (2005) pointed out that these two gyres are observed in the rectangular-shaped side-cavity zone. These gyres are formed in the present LES, irrespective of vertical elevation, and their scale and vortex core positions are almost constant in the vertical direction. Quite similar results are obtained in the PIV data, and it is thus found that the present three-dimensional calculation can reproduce the horizontal mean velocity fields very well.

Although it is difficult to define the core position of the SG in the LES result at $y / H=0.2$, the core positions of the PG and SG are very close between the measured and the calculated data. Further, we estimate a boundary between the PG and SG, considering the longitudinal position where there is zero streamwise velocity, i.e., $U=0$ at the side wall in the embayment, $z / B_{w}=0$. This boundary appears in the ranges $x / B_{w}=1.0$ to 1.5 in both the LES and the PIV. Thus, the present LES is a useful tool to calculate the horizontal gyre structure in the open-channel flow with the side cavity.

\subsection{Mean velocity vectors in vertical plane}

Figure 3 compares the distribution of mean velocity components $(U, V)$ in the vertical planes for the LES and PIV, in which three spanwise positions, $z / B_{w}=0.1,0.5$, and 0.9 were chosen. These positions correspond to 1) near sidewall in the embayment, 2) center line in the embayment, and 3) near boundary between the main channel and the embayment. At $z / B_{w}=0.1$, there exist a positive streamwise current and a negative one in the upward side and the downward side, respectively, and they form SG and PG significantly. It is furthermore found that their boundary appears at about $x$ / $B_{w}=1.0$, where upward flows are generated. At $z / B_{w}=0.5$, in $x / B_{w}=2.5$ to 3.0 , a clockwise circulation with spanwise axis is observed near the bottom, and the above-mentioned boundary of 
PG and SG emanates at $x / B_{w}=1.5$ to 2.0. In contrast, in $z / B_{w}=0.9$, small-scale circulations are formed in the bottom region of the both the upstream and the downstream sides. The PG/SG boundary exists at $x / B_{w}=0.5$. Note that these two-dimensional features could be observed in the both the calculation and the experiment. These results imply that the present calculation could reproduce not only typical horizontal circulations but also small-scale vortices with spanwise axis and the related upward currents. Most previous studies supposed that three-dimensionality is negligible. However, upward and downward currents are generated significantly in the vertical plane and wall regions. Thus, not much is known about the three-dimensional structure within the side cavity.

\subsection{Mean velocity vectors in cross-sectional plane}

Figure 4 shows the mean calculated velocity vectors $(V, W)$, in which the contour line indicates the mean spanwise velocity $W$. It is generally difficult to obtain the structure of these secondary currents by the PIV technique, and thus numerical simulation plays a significant role in the scientific investigations of such currents. At $x / B_{w}=0.2$ near the upstream end of the cavity, the negative spanwise flows prevail near the side wall; in contrast, strong positive lateral currents are observed near the main-channel/embayment junction. The former currents form the SG, as shown in Fig. 2. At $x / B_{w}=0.5$, a zone of negative lateral flows is reduced, whereas a zone of positive flows is extended. Furthermore, longitudinal vortices are generated in the free-surface and bottom regions at the main-channel/embayment interface. At $x / B_{w}=1.0$, these vortices become larger, and strong positive spanwise flows toward the main channel are observed in the center zone, $z / B W=0.5$. There is no flow pattern corresponding to the $\mathrm{SG}$ observed at $x / B_{w}=0.2$. We can see the longitudinal vortices near the junction of $z / B_{w}=1.0$. At $x / B_{w}=2.5$, where the downstream of the PG core is situated, the longitudinal vortices become invisible; at the same time the negative lateral currents prevail in the center zone, contrary to the results of $x / B_{w}=1.0$ and 2.0. Also at $x / B_{w}=2.8$ near the downstream-end, negative lateral currents are observed in the center zone. These results suggest that the cross-sectional flow pattern varies significantly in the streamwise direction, and the lateral transport direction of the mass and momentum depend on streamwise and spanwise positions accompanied by the strong three-dimensional structure.

\subsection{Spatial variations of mean streamwise and spanwise velocity components}


Figure 5 shows the spanwise variations of mean streamwise velocity $U$ at $y / H=0.8$. The result is normalized by a maximum streamwise velocity in the calculated region, $U_{\max }$. The calculated data correspond well with the measured data, except for the main-channel region near the upstream end, $x / B_{w}=0.1$. A thickness of boundary layer becomes larger in the ranges from $x / B_{w}=0.1$ to 2.0, where counter flows $(U<0)$ are accelerated close to the side wall, $z=0$. A zero-cross point situated in the main-channel/embayment boundary at $x / B_{w}=0.1$ is shifted to the within embayment at $x /$ $B_{w}=2.0$. At this position, the counter flow is decelerated, and then the zero-cross point returns toward the main-channel/embayment interface.

Figure 6 shows the longitudinal variation of the normalized spanwise velocity component at the interface, in which the vertical position is quite the same as in Fig. 5, i.e., $y / H=0.8$. Of particular significance is that the prevailing zones of inflow $(W<0)$ and outflow $(W>0)$ are classified clearly. That is to say, we can see the lateral flows from $x / B_{w}=0$ to 2.0; in contrast, counter direction currents are observed from $x / B_{w}=2.0$ to 2.8. The present LES could reproduce this property. Unfortunately, there is a large gap between simulation and experiment for the negative peak value of spanwise velocity around at $x / B_{w}=2.5$.

\subsection{Horizontal distributions of Reynolds stress}

Figure 7 shows the horizontal distribution of Reynolds stress $-\overline{u w}$ at $y / H=0.6$ and 0.8 . The results are normalized by $U_{\max }$. Large positive Reynolds stress is formed along the interface between the mainstream and cavity. These high Reynolds stress zones are relevant to small-scale coherent vortices induced by shear instability. The width of these zones increases in the longitudinal direction, irrespective of the vertical elevation. This spatial property resembles the common feature of the mixing layer phenomenon that promotes mass and momentum exchanges through the interface. Note that the maximum Reynolds stress emanates at $x / B w=1.5$ in the both the simulation and the experiment. This result suggests that the present LES is very reliable in revealing the hydrodynamics in and around the embayment. The Reynolds stress decreases in the downstream side, and thus it may be concluded that maximum exchange through the interface appears at $x / B w$ $=1.5$. The high Reynolds stress zone enters the cavity zone in the downstream side, and it is possible that this mixing layer gives some impacts on the formation of the PG and the SG.

The present LES could obtain valid data that agree well with the measured data. Thus, from a comparison with the measured results, the standard LES can be seen as being capable of 
reasonably reproducing not only mean fields but also turbulence fields.

\section{INSTANTANEOUS VELOCITY FIELDS IN THE EMBAYMENT AND RELATED MOMENTUM TRANSFER PROPERTIES}

\subsection{Time-series of instantaneous secondary currents}

Figure 8 shows the calculated time variation of instantaneous velocity vectors $(\widetilde{v}, \widetilde{w})$ in the cross-sectional plane at $x / B_{w}=1.0$. As shown in the result of $x / B_{w}=1.0$ in Fig. 4 , the mean inward flows through the boundary, $z / B_{w}=1.0$, prevail near the free surface and the embayment bottom. In contrast, the outward flows toward the mainstream appear near the free-surface at $t=0.4 \mathrm{~s}$, in the instantaneous velocity fields. The mean spanwise velocity component in the mid-depth region of $y$ / $H=0.5$ is much smaller than that observed in the free-surface and bottom regions. Thus, the time-averaged results make us believe that a significant momentum transfer takes place in the free-surface and bottom regions. Note, however, that strong inward and outward currents appear alternatively in the main-channel/embayment interface, and thus the momentum exchanges happen more intensively in the mid-depth region than in other depth regions. Therefore, we should consider not only the time-averaged flow pattern but also the instantaneous velocity fields for a deep investigation of mass and momentum exchanges between the main-channel stream and the cavity zone.

\subsection{Quadrant conditional sampling analysis}

Nezu and Nakagawa (1993) and Sanjou and Nezu (2011) conducted quadrant condition sampling techniques to quantitatively evaluate bursting properties in open-channel flows. In the present subsection, this mathematical technique is introduced to reveal vertical profiles of the instantaneous momentum transfer. The quadrant conditional Reynolds stress components are defined in the following form:

$$
R S_{i}=(\overline{u w})^{-1} \lim _{T \rightarrow \infty} \int_{0}^{T} u w I_{i} d t \quad(i=1,2,3,4)
$$

In the actual calculation process, $T$ is given by the finite sampling time.

If $(u, w)$ exists in a quadrant $i$, then $I_{i}=1$, otherwise $I_{i}=0$. Each quadrant of $(u, w)$ corresponds to the following coherent events: 
$i=1, u>0, w>0$ : interaction (outward motion)

$i=2, u<0, w>0$ : ejection (outward motion)

$i=3, u<0, w<0$ : interaction (inward motion)

$i=4, u>0, w<0$ : sweep (inward motion).

$R S 1$ and $R S 2$ correspond to the outward flow toward the mainstream, and $R S 3$ and $R S 4$ correspond to the inward flow toward the cavity. Figure 9 shows calculated longitudinal variations of $R S i$ at the boundary, $z$ / $B=1.0$. A common feature that may be observed is that $R S 2$ and $R S 4$ are much larger than $R S 1$ and $R S 3$, which means the interaction events are not so significant compared with the ejections and the sweeps. The same properties are observed in various two-dimensional boundary and mixing layers, such as wall turbulence in smooth bed, vertical mixing layer in vegetated canopy open-channel flows, and horizontal mixing layer in compound channels (e.g., Nezu \& Sanjou 2008 and Sanjou \& Nezu 2011). In the bottom region of $y / H=0.2, R S 4$ (ejection) prevails compared with RS2; this implies that a high-speed fluid parcel $(u>0)$ inrushes to the embayment from the mainstream. At $y / H=0.4, R S 2$ is relatively close to $R S 4$, whereas, $R S 4$ becomes comparatively more dominant than $R S 2$ at $y / H=0.6$, suggesting that the outward flow with the low-speed momentum emanates together with high probability.

A similar result is also observed at $y / H=0.8$.

Figure 10 shows the predicted vertical profiles of $R S i$ normalized by $U_{\max }$, i.e.,

$\left(\lim _{T \rightarrow \infty} \int_{0}^{T} u w I_{i} d t\right) / U_{\max }^{2} R S 2$ is very close to $R S 4$, and they increase with the development of the horizontal mixing layer in the range from $x / B_{w}=0$ to 0.5 for all depth layers. This result suggests that the two-dimensional structure may be kept near the upstream end of the embayment. The SG core appears at $x / B_{w}=0.5$, as shown in Fig. 2, and it is therefore expected that the contribution of the SG to the momentum exchange is relatively small. In the downstream zone from $x / B_{w}=0.5$ to 3.0, significant differences are observed except for $y / H=1.0$. At $y / H=0.2, R S 2$ is larger than $R S 4$, and thus inward motions are produced. In contrast, at $y / H=0.4,0.6$, and 0.8 , outward motions from the mainstream prevail. In particular, at $y / H=0.6$, there exists a distinct peak of $R S 2$ at $x / B w=2.0$, where the PG core is observed. Thus, the PG may play important roles in the momentum transfer from the mainstream toward the embayment. Although, at $y / H=1.0$, there are complicated distributions for $R S 2$ and $R S 4$, the difference between them is much smaller than the 
other vertical elevations. Consequently, a complex three-dimensional structure is formed instantaneously, accompanied by the transfer of high-speed and low-speed fluid parcels in the range from $x / B w=0.5$ to 3.0.

\subsection{Relation between the lateral momentum transfer and vertical current}

Figure 11 shows the calculated distribution of cross-sectional Reynolds stress $-\overline{v w}$ in a vertical plane placed on the junction, $z / B_{w}=1.0$. The negative zone is situated in the mid-depth region corresponding to the ejection-prevailing region $(\widetilde{w}>0)$. Therefore, the ejection event is generated together with instantaneous upward current $(\tilde{v}>0)$. Positive distributions are seen in the free-surface and bed regions.

The quadrant conditional sampling Reynolds stress for the cross-sectional component $\overline{v w}$ could be given in the following equation in the same manner as Eq. (10):

$$
R S c_{i}=(\overline{v w})^{-1} \lim _{T \rightarrow \infty} \int_{0}^{T} v w I_{i} d t \quad(i=1,2,3,4)
$$

in which

$i=1, w>0, v>0$ : (outward motion with upward current)

$i=2, w<0, v>0$ : (inward motion with upward current)

$i=3, w<0, v<0$ : (inward motion with downward current)

$i=4, w>0, v<0$ : (outward motion with downward current).

$R S c 1$ and $R S c 4$ correspond to the outward momentum. Thus, their ratio $R S c 1 / R S c 4$ could be a criterion to define which prevails, the upward current or the downward current. $R S c 2$ / $R S c 3$ could be 1 for the inward momentum transfer; when this value is larger than 1, it implies that the upward current is more dominant than the downward current. Figure 12 shows their longitudinal profiles at the main-channel/embayment interface. At $y / H=0.2$, the generation possibility of upward current is comparatively high during the inward momentum transfer near the upstream and downstream sides of the embayment. The generation possibilities of the upward and downward flows are almost same in the center region from $x / B_{w}=1.0$ to 2.0. RSc $1 / R S c 4$ is found to be larger than 1 and $R S c 2$ / RSc3 is smaller than 1 in the almost streamwise region at $y / H=0.4$. The outward motion and the 
inward motion are generated together with the upward and downward currents, respectively. At $y$ / $H=0.6$, the region of $R S c 1 / R S c 4>1$ is very remarkable, and $R S c 2 / R S c 3$ is smaller than 1 in the entire streamwise region. This suggests that the outward transfer with the upward current and the inward transfer with the downward current are significant at the present elevation. At $y / H=0.8$, the outward transfer is often accompanied by the downward current in the upstream side, $x / B_{w}$ $<1.5$. In contrast, the inward transfer with downward current prevails except in the upstream and downstream boundary regions. At the free surface, $y / H=1.0$, we can see the inward transfer with the upward motion.

\section{PHENOMENOLOGICAL FLOW MODEL}

Figure 13 shows the three-dimensional phenomenological flow model developed using the above-mentioned numerical results, in which there are mean horizontal gyres and instantaneous momentum transfer with high probability. Within the cavity with an aspect ratio of 3.0, two kinds of horizontal gyres are generated with counter rotations, and their vertical variation is comparatively small compared with the horizontal structure, as shown in Fig. 2. Contrary to the time-averaged gyres, there exist complicated three-dimensional properties for the lateral momentum transfer through the junction between the mainstream and the side cavity. The vertical motions are noted significantly within the canopy, as shown in Fig.3. A combination of upward and downward currents produces a stagnation zone, and spanwise vortices are formed in the both of the upstream and downstream edges.

The comparison of $R S 2$ and $R S 4$ helps us know the prevailing turbulence transport in the mainstream/embayment boundary as shown in Figs. 9 and 10. In the bottom layer, inflow momentum is frequently produced with the exception of the near-upstream end, $x / B_{w}=0$. In the mid-depth layer, the outward momentum transfer prevails remarkably. Further, the inflow and the outflow appear alternatively in the free surface. The conditional analysis of cross-sectional Reynolds stress indicated in Fig. 12 allows us to understand the relationship between the momentum transfer and the vertical motions. That is to say, it is highly possible that these lateral transfers are generated together with the vertical currents, irrespective of the depth direction.

\section{CONCLUSIONS}

A large eddy simulation was conducted for the open-channel flows with side-cavity zone in order to 
reveal three-dimensional flow patterns and related momentum exchanges through the mainstream/embayment interface. In the present study, PIV measurements were also carried out to examine the calculation accuracy. The main findings are as follows:

1) Two kinds of horizontal gyres are generated in the whole depth region: the primary gyre induced by the velocity shear between main channel and embayment, and the secondary gyre observed near the primary gyre. This interesting result coincides with previous studies. These gyres can be reproduced by the present LES, and their length scale and vortex core positions agree well with those observed in the PIV measurements.

2) The existence of the horizontal gyres has striking effects on the cross-sectional flow field. The calculated flow pattern varies significantly in the streamwise direction, and the lateral transport direction of the mass and momentum depend strongly on the streamwise and spanwise positions.

In the vertical plane, there are small-scale vortices with spanwise axis and related upward currents. Although most previous studies supposed three-dimensionality to be negligible, upward and downward currents are surely generated in the PG/SG interface and wall regions.

3) Strong inflows and outflows are generated alternatively in the junction between the mainstream and the side cavity. Further, it was found that the momentum exchanges are observed most frequently in the mid-depth layer. Therefore, it is important for the investigation of the momentum transfer phenomenon to consider not only the time-averaged flow pattern but also the instantaneous velocity fields for a deep investigation of mass and momentum exchanges between the main-channel stream and side dead-water zone.

4) A phenomenological flow model was proposed on the basis of the results of the conditional sampling analysis. Of particular significance is that a complex three-dimensional structure is formed instantaneously, accompanied by the transfer of high-speed and low-speed fluid parcels. In particular, inward transfer is often observed near the bottom. In the mid-depth layer, outward flows are more significant; in contrast, the inflow and the outflow appear alternatively near the free surface. 


\section{ACKNOWLEDGEMENT}

The present study was carried out with financial support from the Research Project Grant-In-Aid for

Scientific Research of Japanese Government (Grant-in-aid for young scientists No. 23760458, Principal Investigator M. Sanjou). The authors gratefully acknowledge this support.

\section{REFERENCES}

Abad, J.D., Rhoads, B.L., Guneralp. I. and Garcia, M.H. (2008). Flow structure at different stages in a meander bend with bendway weirs. J. Hydraulic Engineering, Vol. 134(8), pp. 1052-1063.

Booij, R. (2004). Laboratory experiments of shallow free surface flows. Shallow Flows Vol.2, Jirka \& Uijttewaal eds., pp.317-323.

Chang, K.S., Constantinescu, S.G. and Park, S. (2006). Analysis of the flow and mass transfer process for the incompressible flow past an open cavity with a laminar and fully turbulent incoming boundary layer. J. Fluid Mech. Vol. 561, pp.113-145.

Chang, K.S., Constantinescu S.G. and Park, S. (2007). The purging of a neutrally buoyant or a dense miscible contaminant from a rectangular cavity. Part II: The case of an incoming fully turbulent overflow. J. Hydraulic Engineering, Vol. 133(4), pp. 373-385.

Constantinescu, G., Sukhodolov, A. and McCoy, A. (2009). Mass exchange in a shallow channel flow with a series of groynes: LES study and comparison with laboratory and field experiments. Environmental Fluid Mechanics, Vol. 9, pp. 587-615.

Hinterberger, C., Frohlich, J. and Rodi, W. (2007). Three-dimensional and depth-averaged large eddy simulations of some shallow water flows. J. Hydraulic Engineering, Vol. 133(8), pp. 857-872.

Hirt, C.W. and Nicholds, B.D. (1981). Volume of fluid method for the dynamics of free boundaries J. Comp. Phys., Vol. 39, pp. 201-225.

McCoy, A., Constantinescu, S.G. and Weber, L. (2008). A numerical investigation of flow hydrodynamics in a channel with a series of groynes. J. Hydraulic Engineering, Vol. 134(2), pp. 157-172.

Moin, P. and Kim,J. (1982). Numerical investigation of turbulent channel flow. J. Fluid Mech. Vol. 118 pp. 341-377.

Nezu, I. and Nakagawa, H. (1993). Turbulence in Open - Channel Flows. IAHR Monograph, 
Balkema.

Nezu, I. and Sanjou, M. (2008). Turbulence structure and coherent motion in vegetated open-channel flows. Journal of hydro-environment research, Vol. 2, pp. 62-90.

Nezu, I. and Sanjou, M. (2011). PIV and PTV measurements in hydro-sciences with focus on turbulent open-channel flows. Journal of hydro-environment research, Vol. 5, pp. 215-230.

Okamoto, T. and Nezu, I. (2010). Large eddy simulation of 3-D flow structure and mass transport in open-channel flows with submerged vegetations. J. of Hydro-environment Res., No. 118, pp. $1-13$.

Sanjou, M. and Nezu, I. (2009). Turbulence structure and coherent motion in meandering compound open-channel flows. Journal of Hydraulic Research, Vol. 47, No. 5, pp. 598-610.

Sanjou, M. and Nezu, I. (2011). Turbulent structure and coherent vortices in open-channel flows with wind-induced water waves. Environmental Fluid Mechanics, Vol. 11, No. 2, pp. 113-131.

Sanjou, M. and Nezu, I. (2010). Large Eddy Simulation of compound open-channel flows with emergent vegetation near the floodplain edge. Journal of Hydrodynamics, Ser.B, Vol. 22, Issue 5, Sup.1, pp. 582-586.

Uijttewaal, W.S.J., Lehmann, D. and van Mazijk, A. (2001). Exchange processes between a river and its groyne fields: Model experiments. Journal of Hydraulic Engineering, Vol. 127(11), pp. 928-936.

Uijttewaal, W.S.J. (2005). Effects of groyne layout on the flow in groyne fields: Laboratory experiments. Journal of Hydraulic Engineering, Vol. 131(9), pp. 782-791.

Valentine, E.M. and Wood, I.R. (1977). Longitudinal dispersion with dead zones. Journal of Hydr. Div. Vol. 103(9), pp. 975-990.

Weitbrecht, V, Socolofsky, S.A. and Jirka, G. (2008). Experiments on mass exchange between groin fields and main stream in rivers. Journal of Hydraulic Engineering, Vol. 134(2), pp. 173-183.

Yossef, M.F.M. and de Vriend, H. (2011). Flow details near river groynes: Experimental investigation. Journal of Hydraulic Engineering, Vol. 137(5), pp. 504-516. 


\section{Notations}

$\widetilde{p} \quad$ instantaneous water pressure

$u \quad$ velocity fluctuation in the streamwise direction

$\tilde{u} \quad$ instantaneous velocity component in the streamwise direction

$v \quad$ velocity fluctuation in the vertical direction

$\widetilde{v} \quad$ instantaneous velocity component in the vertical direction

$w \quad$ velocity fluctuation in the spanwise direction

$\widetilde{w} \quad$ instantaneous velocity component in the spanwise direction

$w^{\prime} \quad$ turbulence intensity in the spanwise direction

$-\overline{u w} \quad$ horizontal Reynolds stress

$-\overline{v w} \quad$ cross-sectional Reynolds stress

B main-channel width

$B_{w} \quad$ spanwise width of side-cavity

$C_{s} \quad$ Smagorinsky constant

H static water depth

$L_{w} \quad$ longitudinal length of side-cavity

$R S_{i} \quad$ quadrant horizontal Reynolds stress

$R S c_{i} \quad$ quadrant cross-sectional Reynolds stress

$U$ time-averaged mean velocity component in the streamwise direction

$V \quad$ time-averaged mean velocity component in the vertical direction

$W \quad$ time-averaged mean velocity component in the spanwise direction

$U_{m} \quad$ bulk-mean velocity in the water current

$U_{\max } \quad$ maximum streamwise velocity in the calculated region

$x \quad$ streamwise coordinate

y vertical coordinate

$z \quad$ spanwise coordinate

$1 / \Delta t \quad$ data sampling rate

$1 / \Delta \delta \quad$ frame rate of high-speed camera

$v \quad$ viscosity

$v_{T} \quad$ SGS eddy viscosity

$\Delta \quad$ SGS filter 


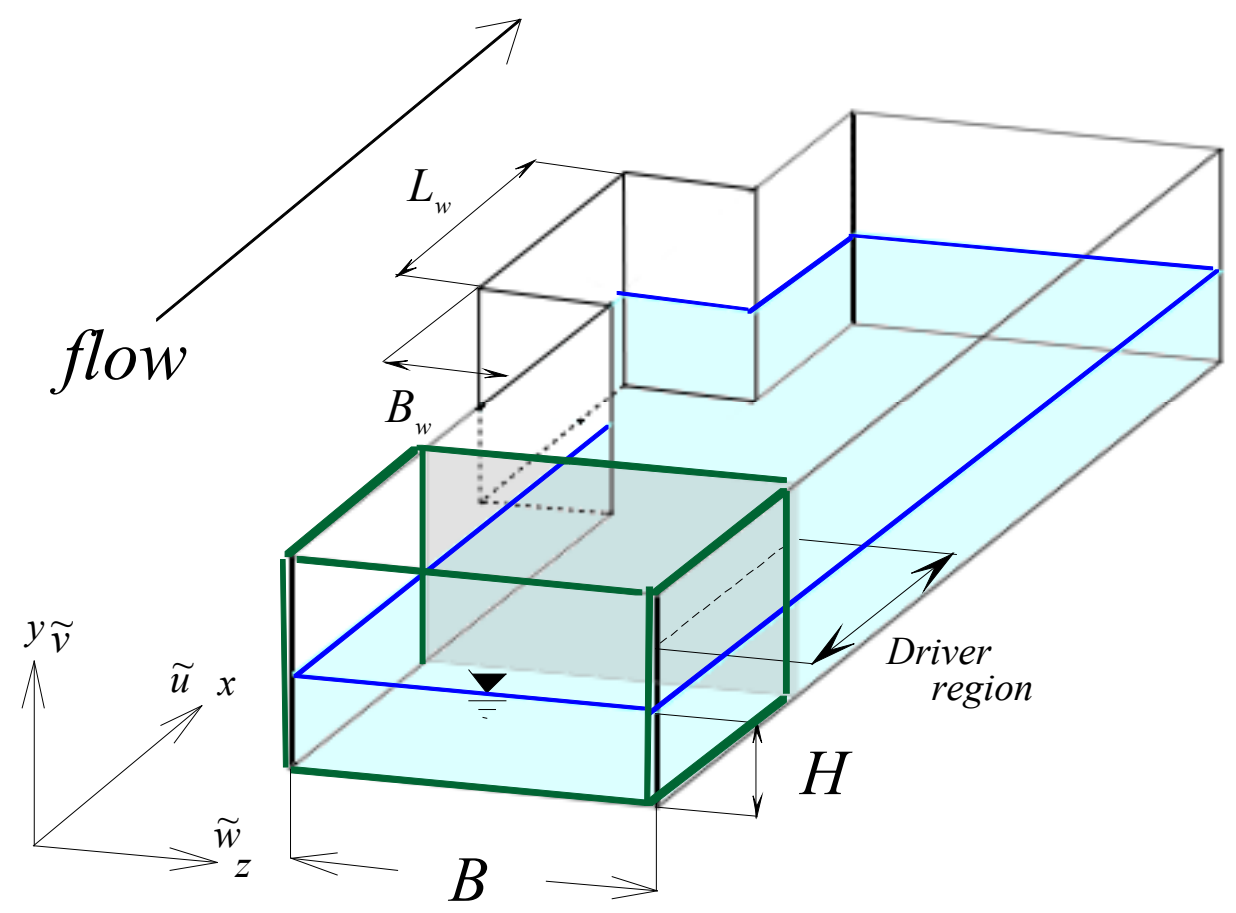

Fig.1 Flow field and coordinate system 
(a) $y / H=0.2$

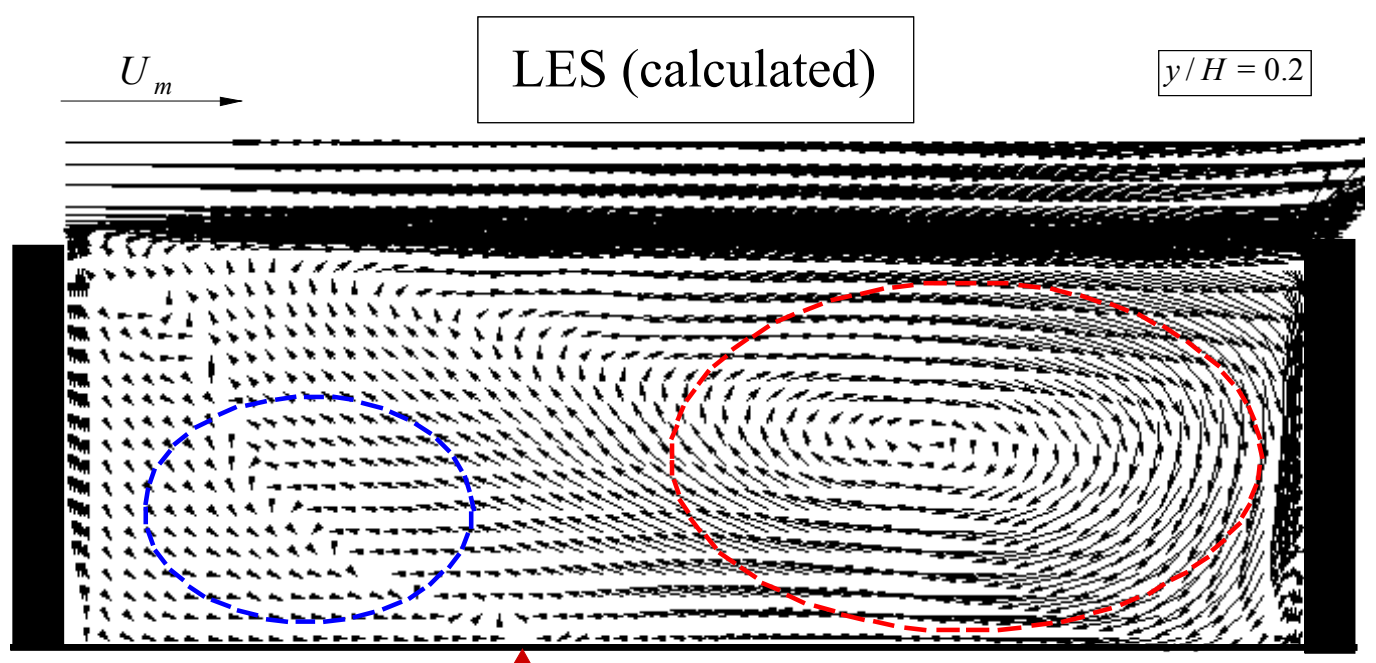

T PG/SG boundary at side-wall

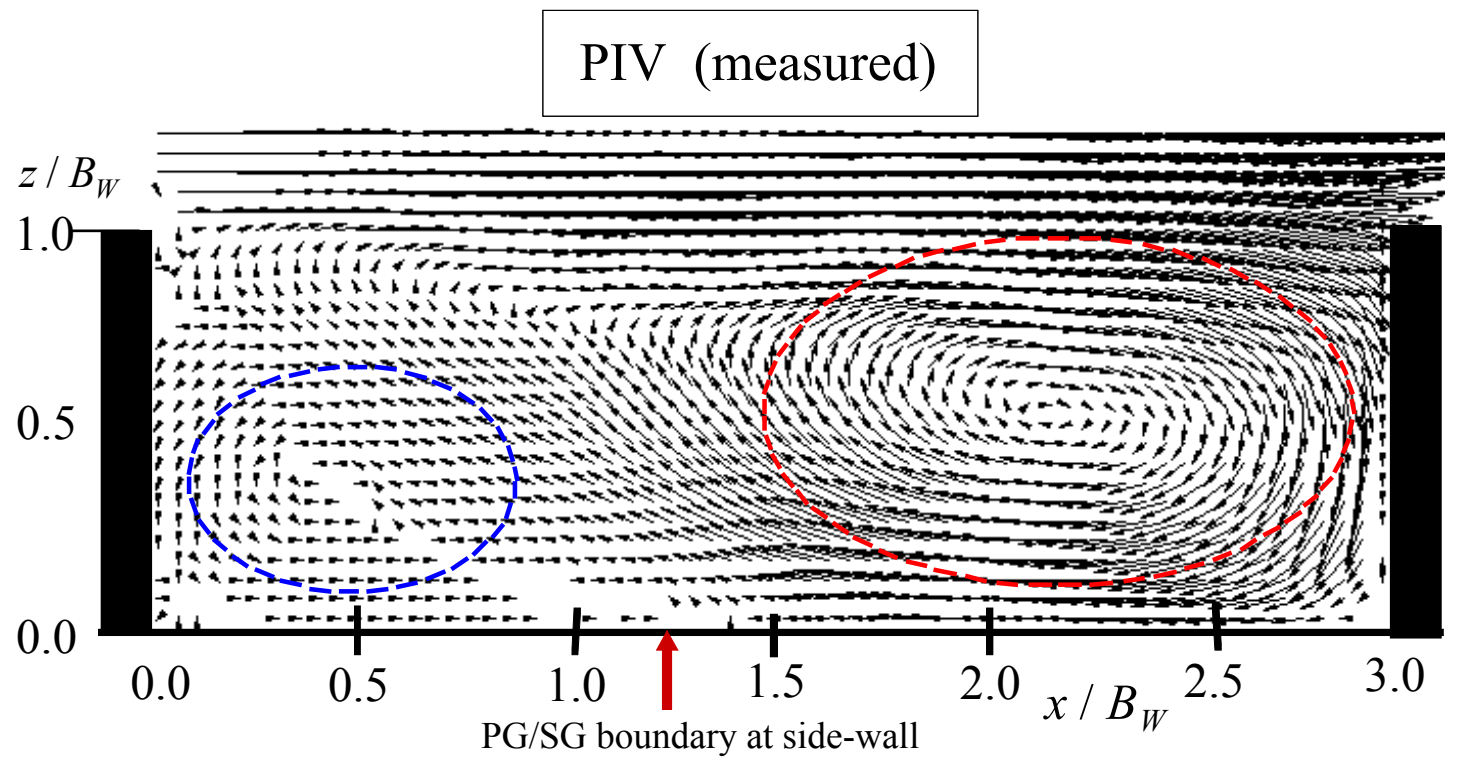


(b) $y / H=0.4$
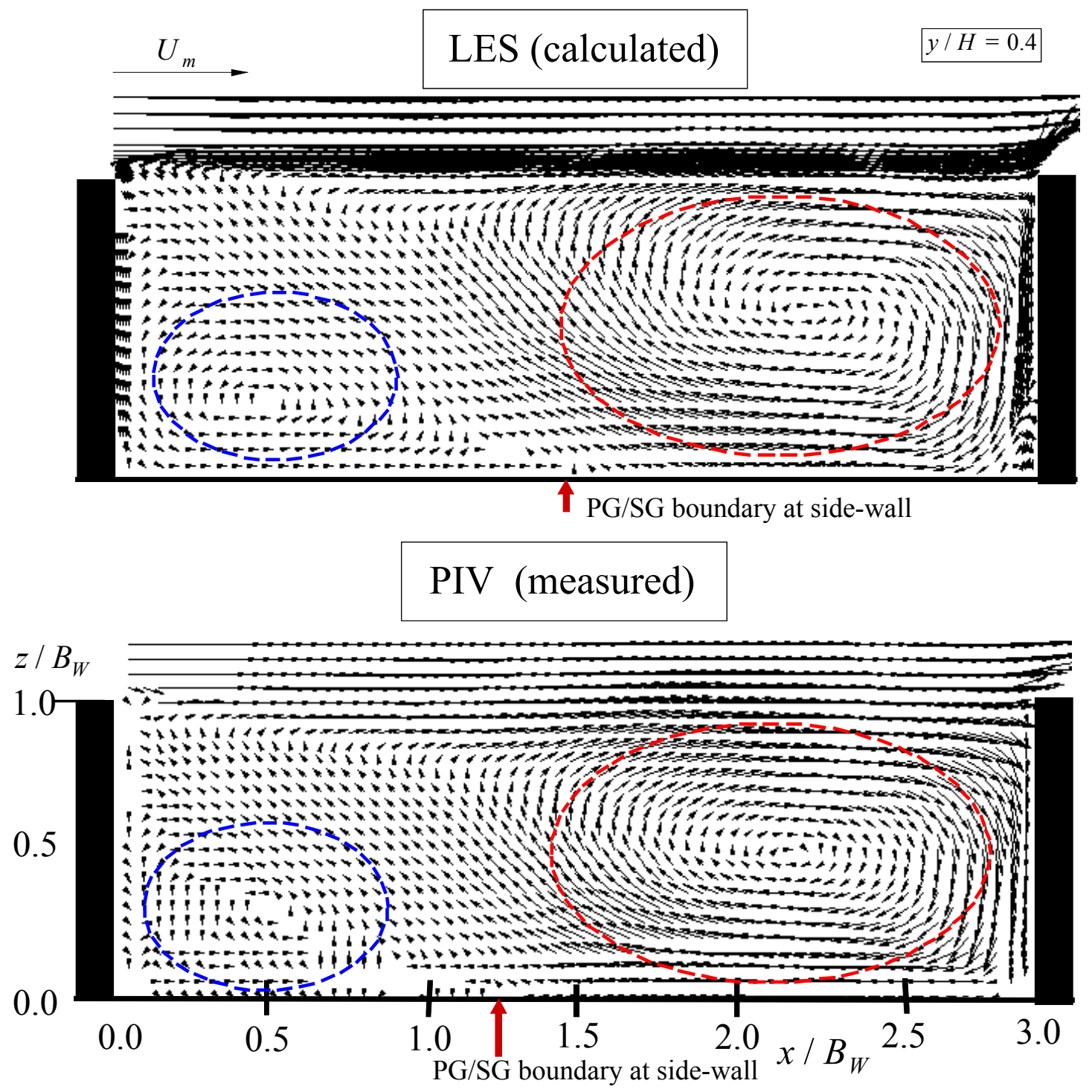
(c) $y / H=0.8$

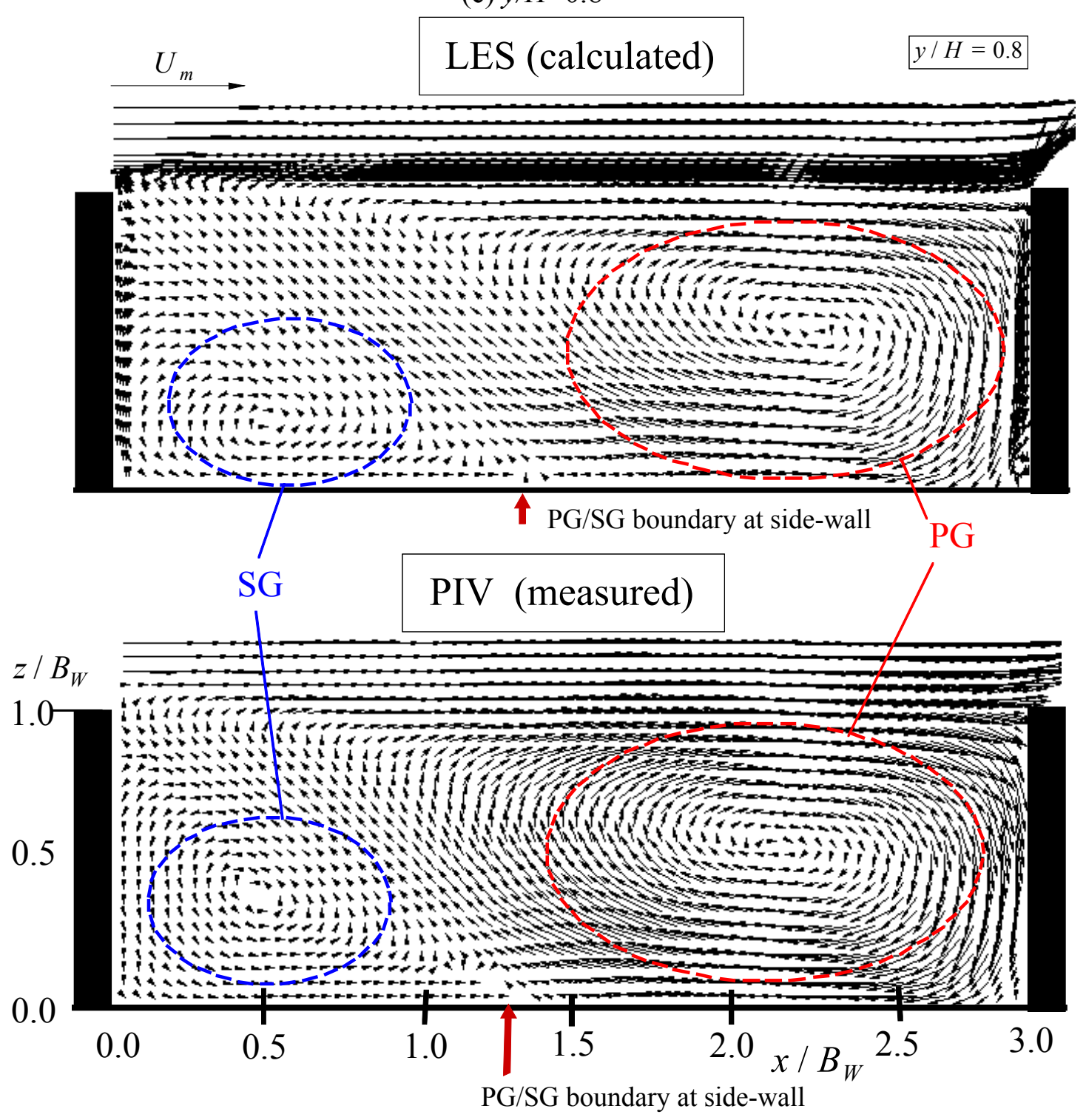

Fig.2 (a) to (c) Mean horizontal veloctity vectors $(U, W)$ of calculated and measured data 
(a) Calculated by LES

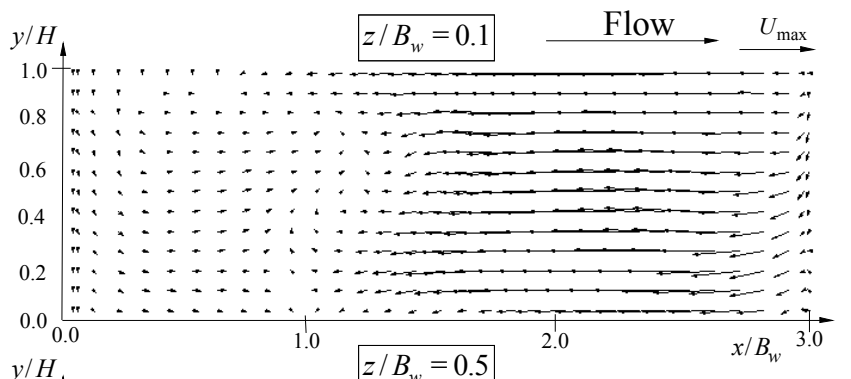

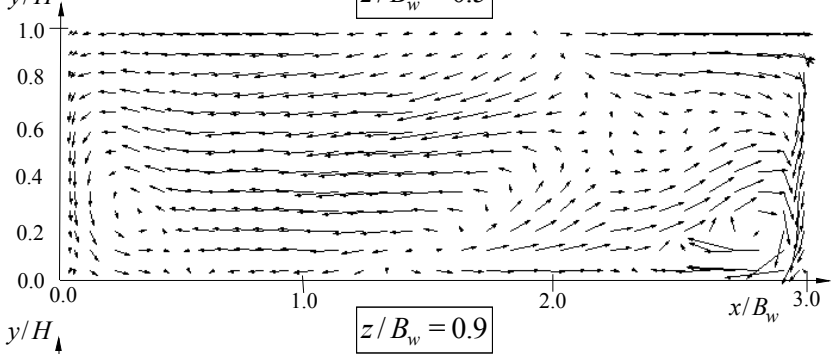
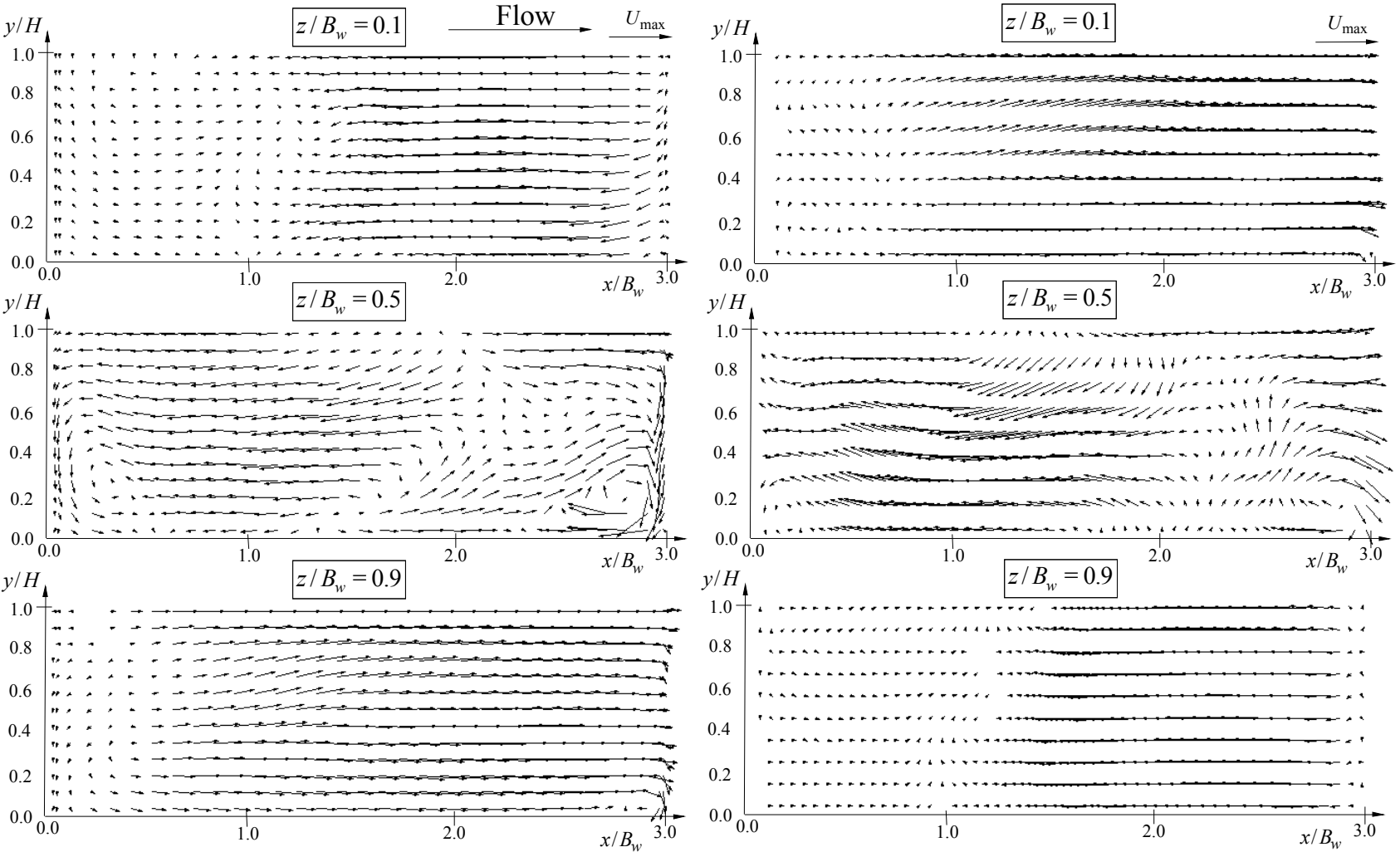

Fig.3 Mean vertical flow patterns $(U, V)$ of calculated and measured data 


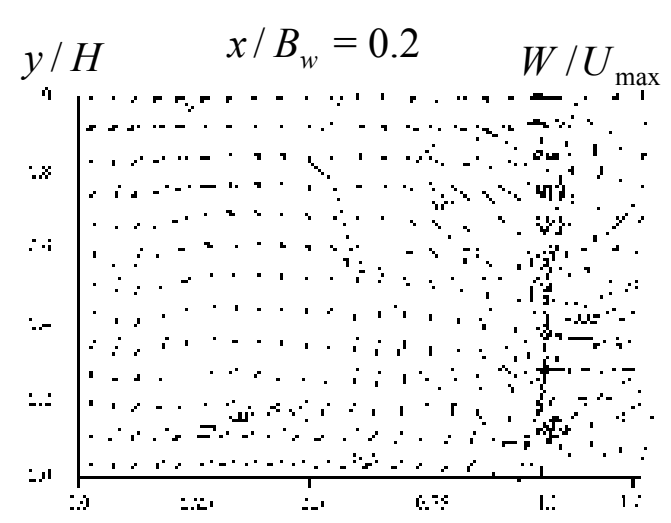

$$
x / B_{w}=0.5
$$

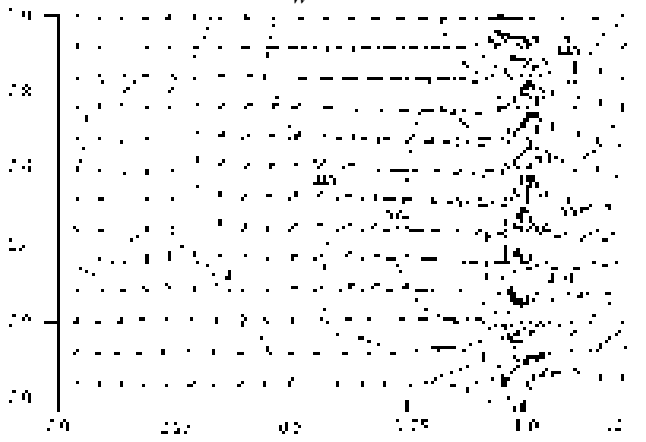

$$
x / B_{w}=1.0
$$

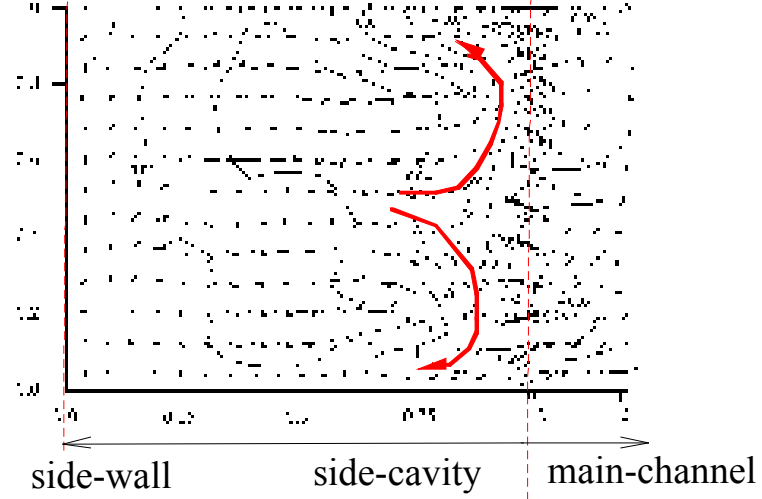

$\underline{0.2 U_{\max }} \quad x / B_{w}=2.0$

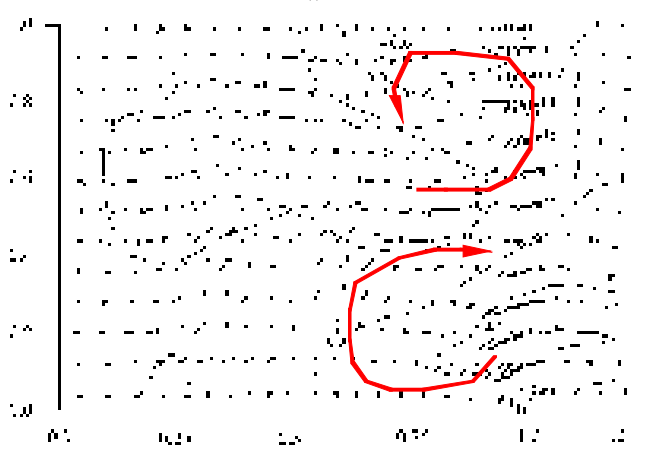

$$
x / B_{w}=2.5
$$

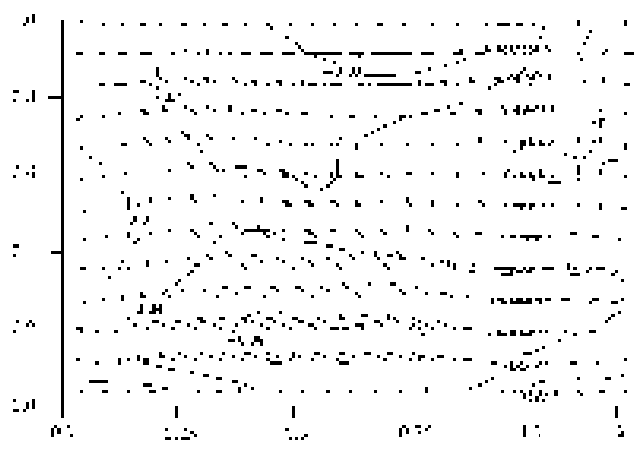

$$
x / B_{w}=2.8
$$

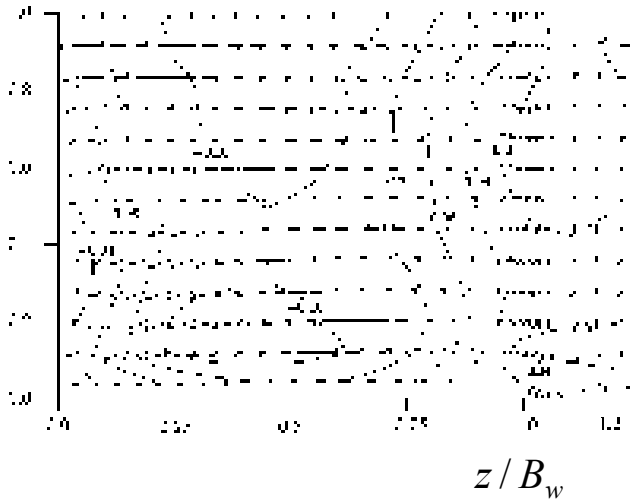

Fig.4 Mean cross-sectional flow patterns $(V, W)$ of calculated data 


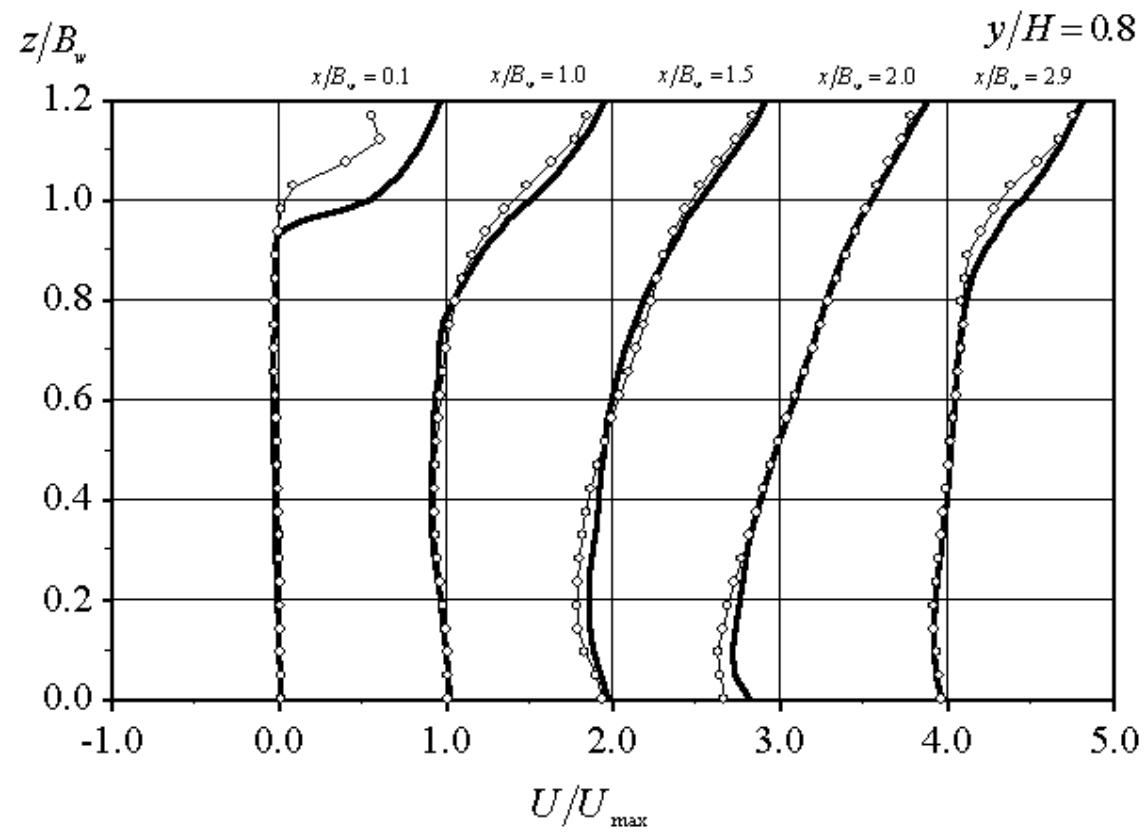

Fig.5 Spanwise profiles of mean streamwise velocity (white circle: PIV and rigid line: LES) 


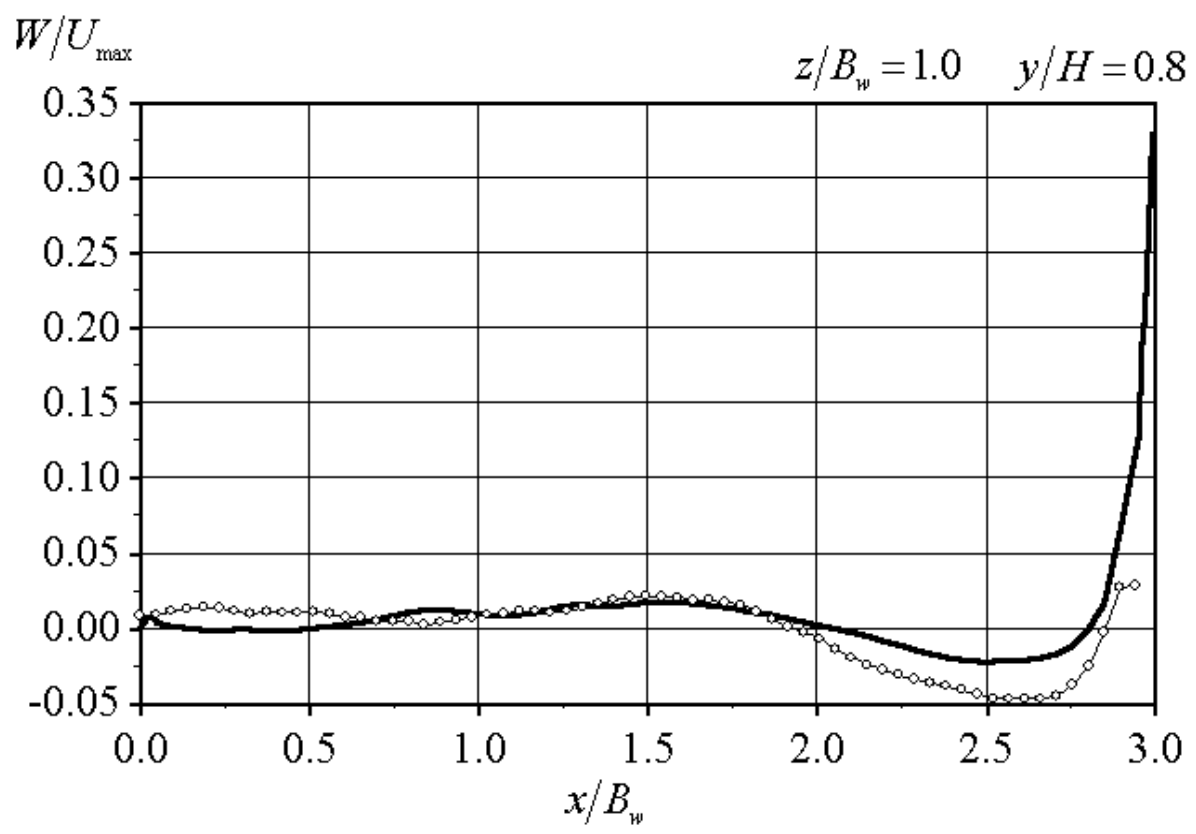

Fig.6 Longitudinal profiles of mean spanwise velocity (white circle: PIV and rigid line: LES) 

(a) LES
(b) PIV
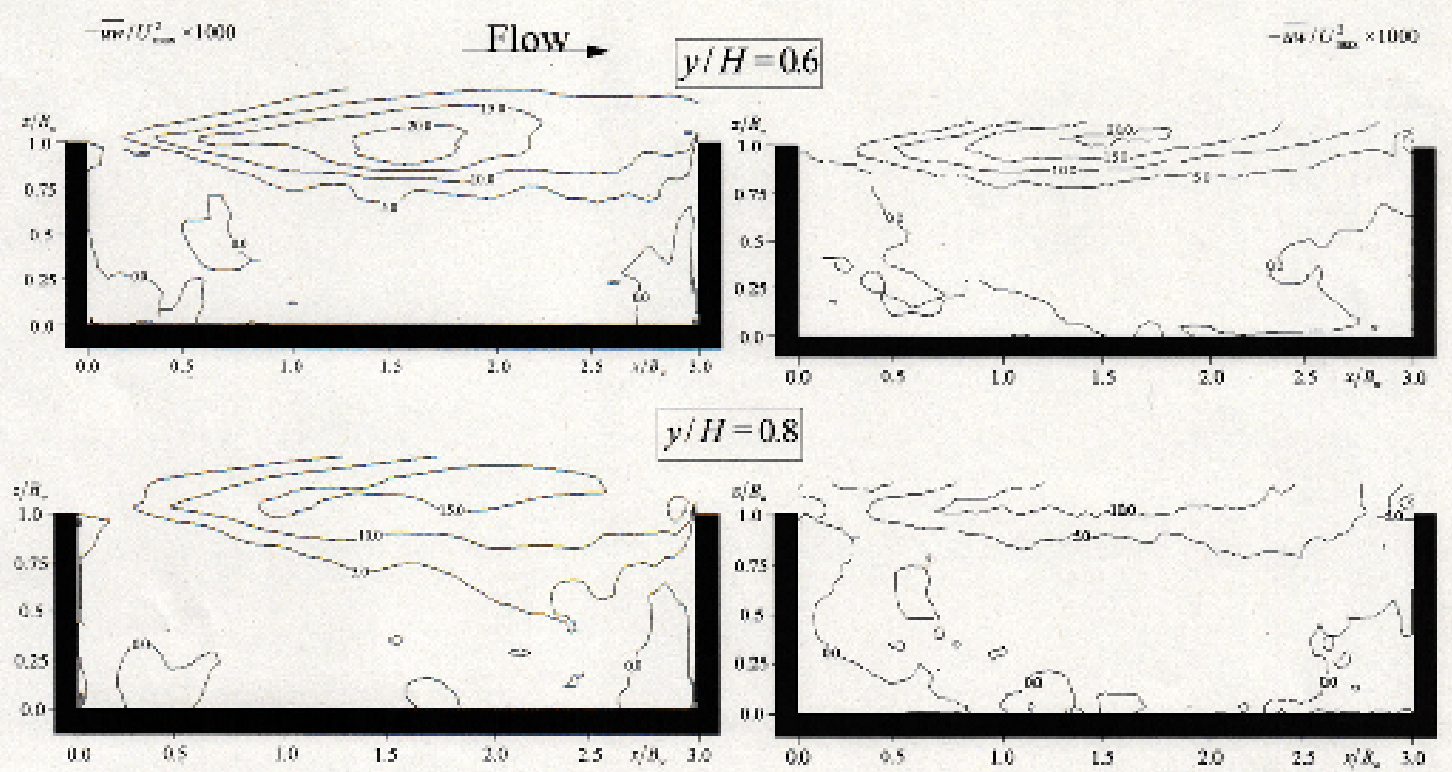

Fig.7 Comparison of calculated and measured distributions of horizontal Reynolds stress 

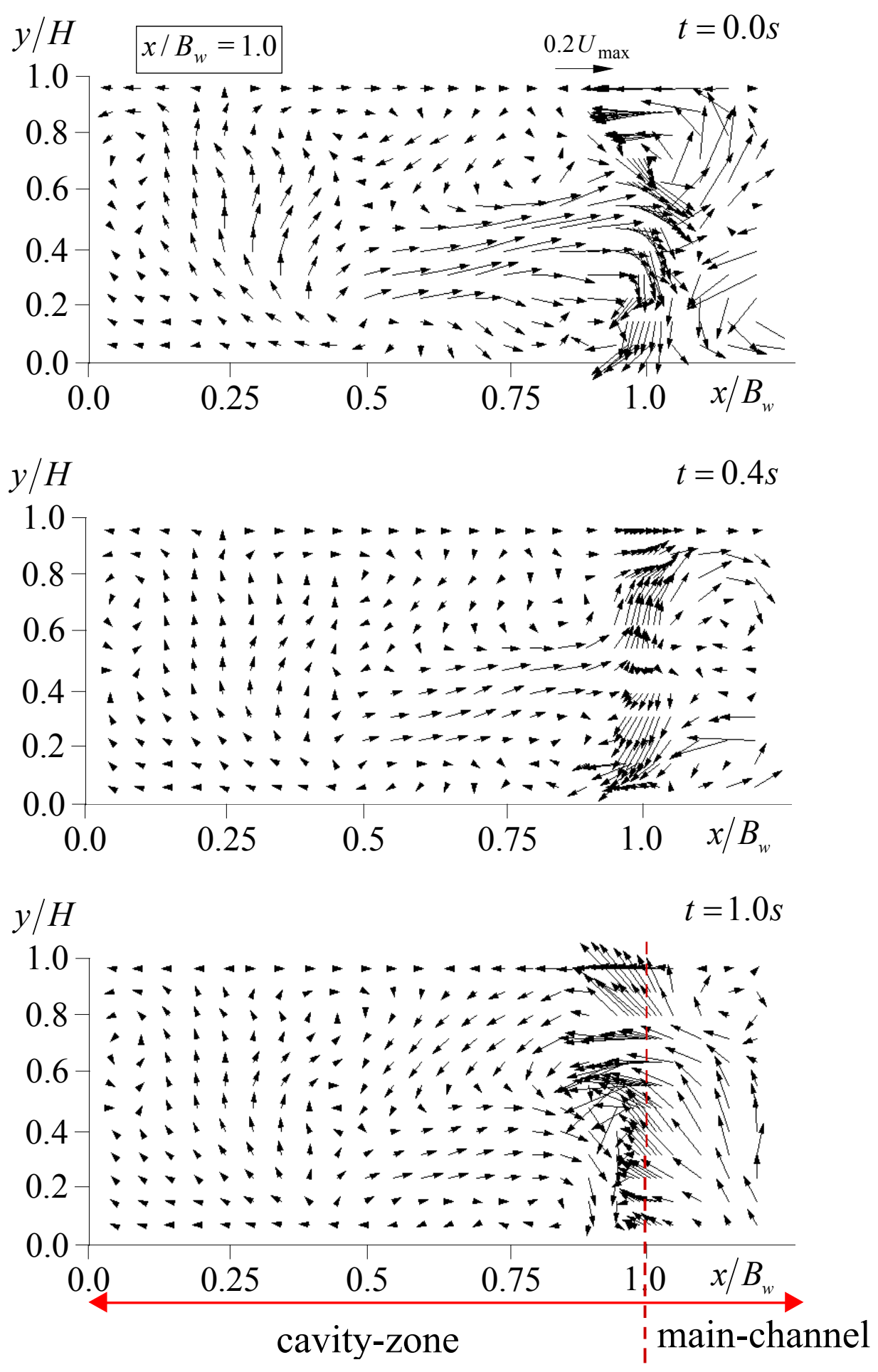

Fig.8 Examples of time-series of instantaneous velocity vectors in cross-sectional plane 

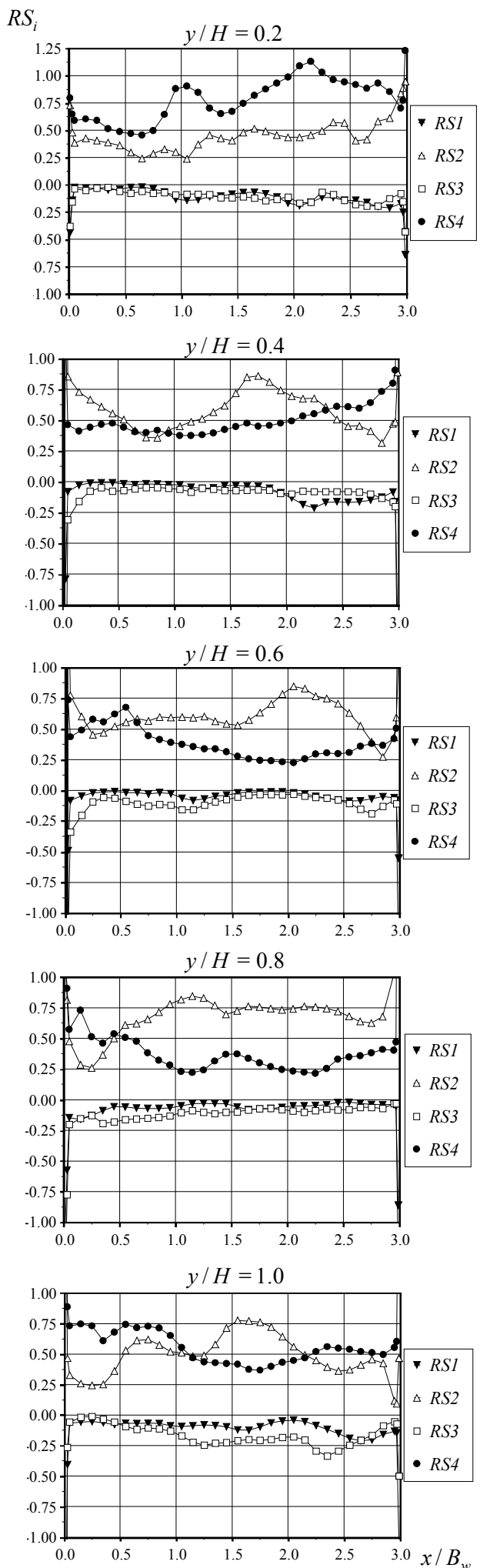

Fig.9 Longitudinal profiles of predicted quadrant Reynolds stress 

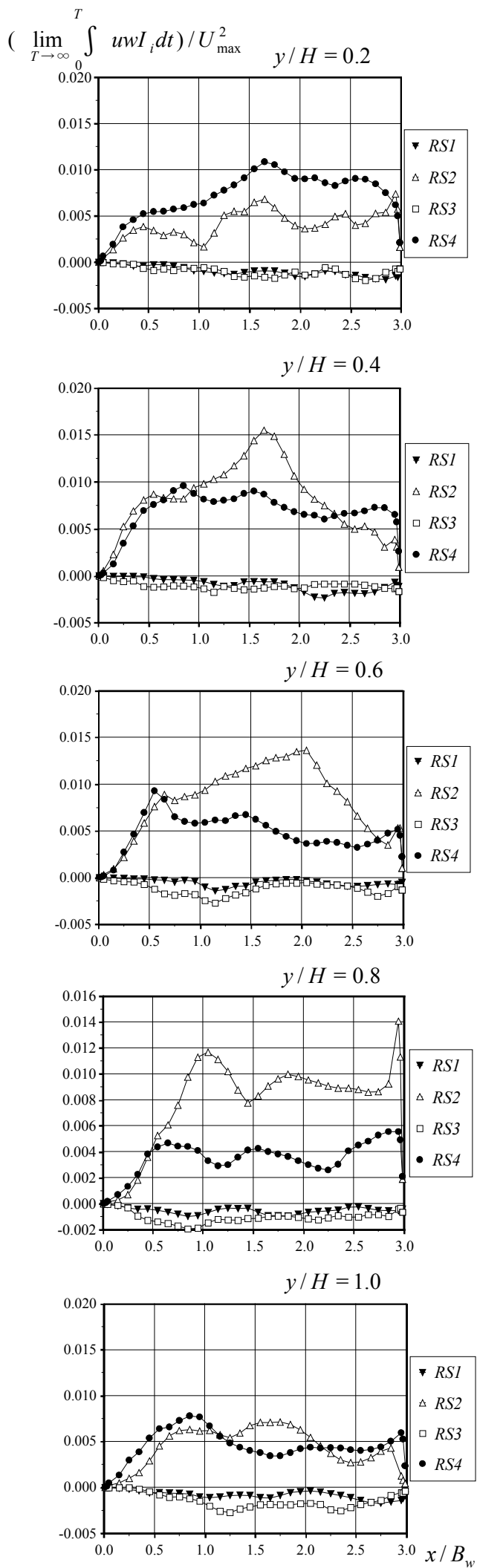

Fig.10 Longitudinal variations of quadrant Reynolds stress normalized by maximum streamwise velocity 


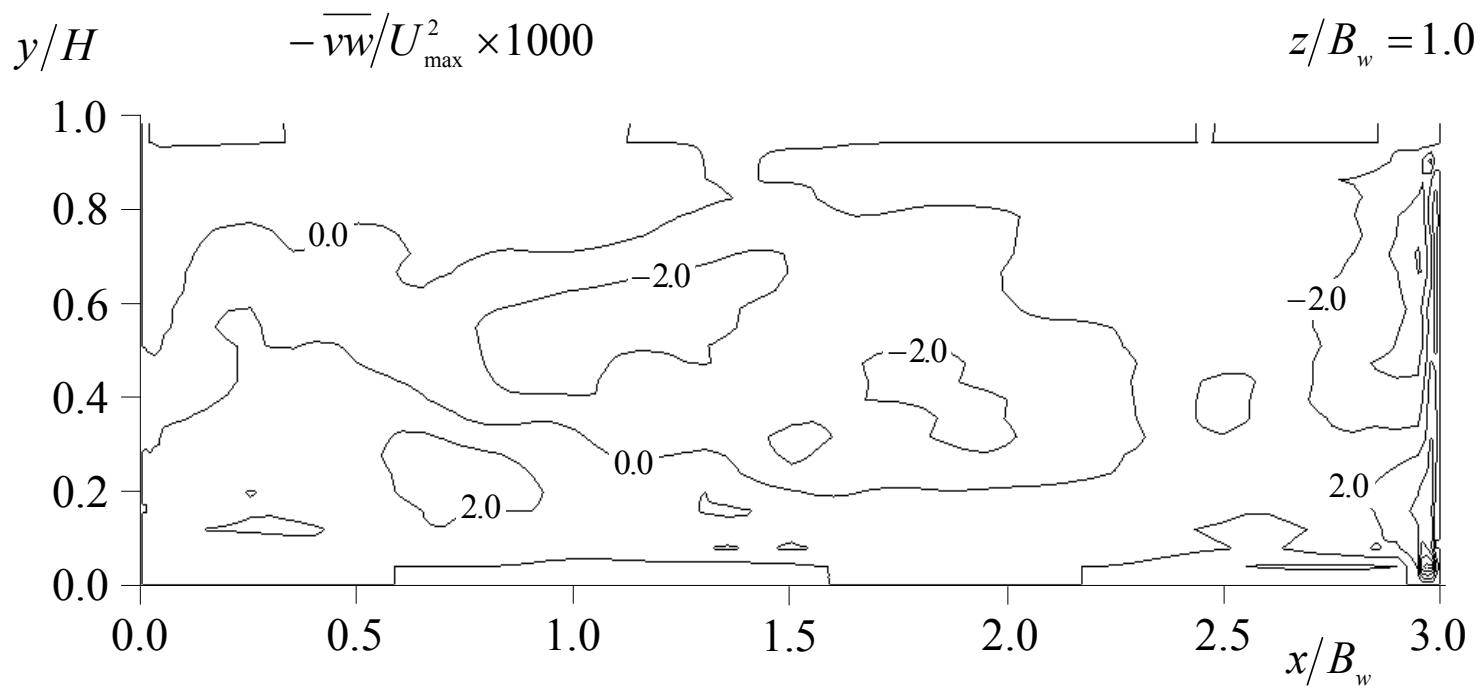

Fig.11 Distribution of calculated cross-sectional Reynolds stress in the vertical plane 
(a)

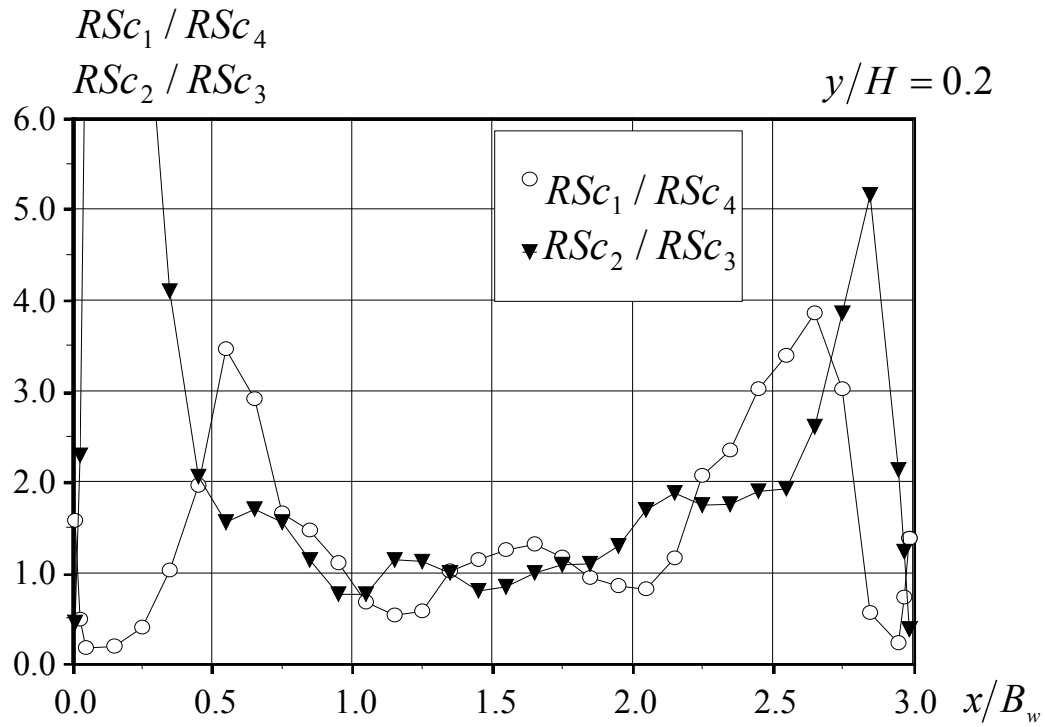

(b)

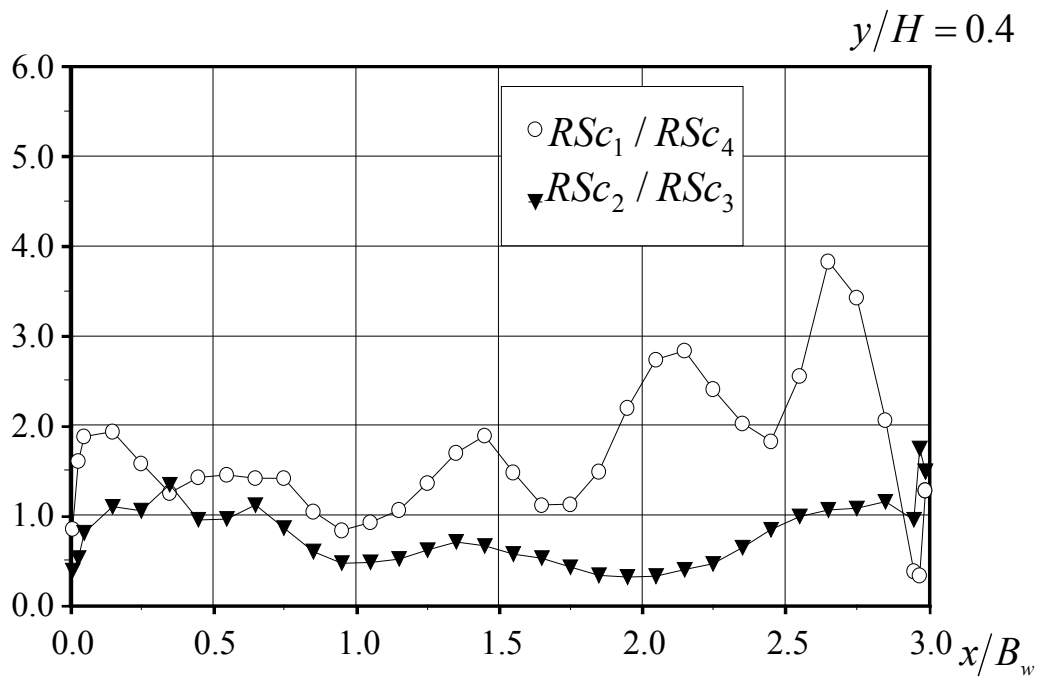

(c)

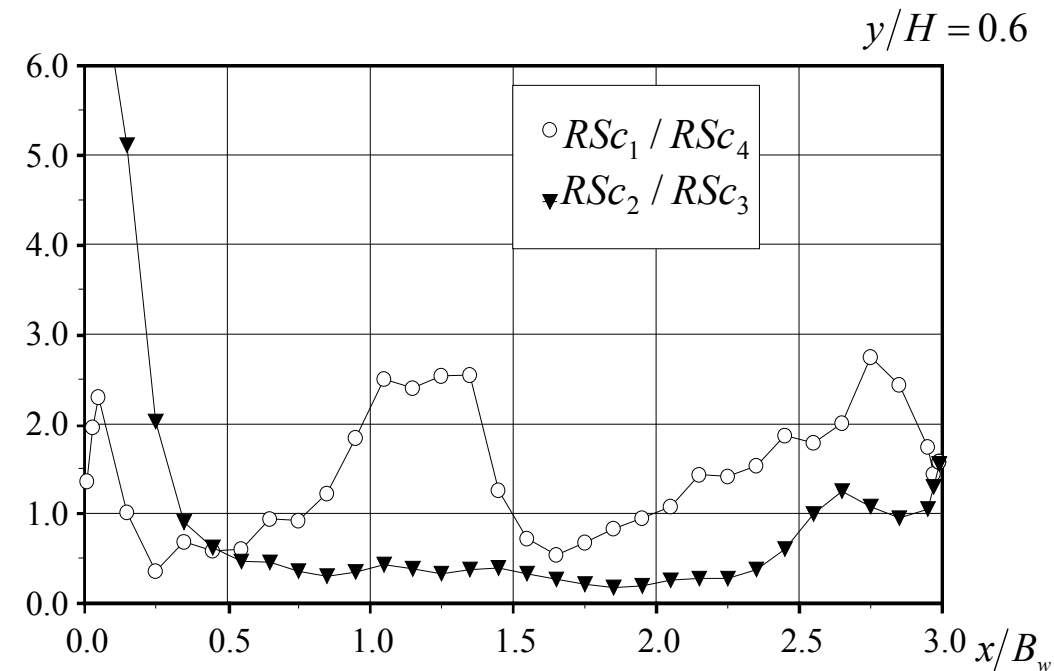


(d)

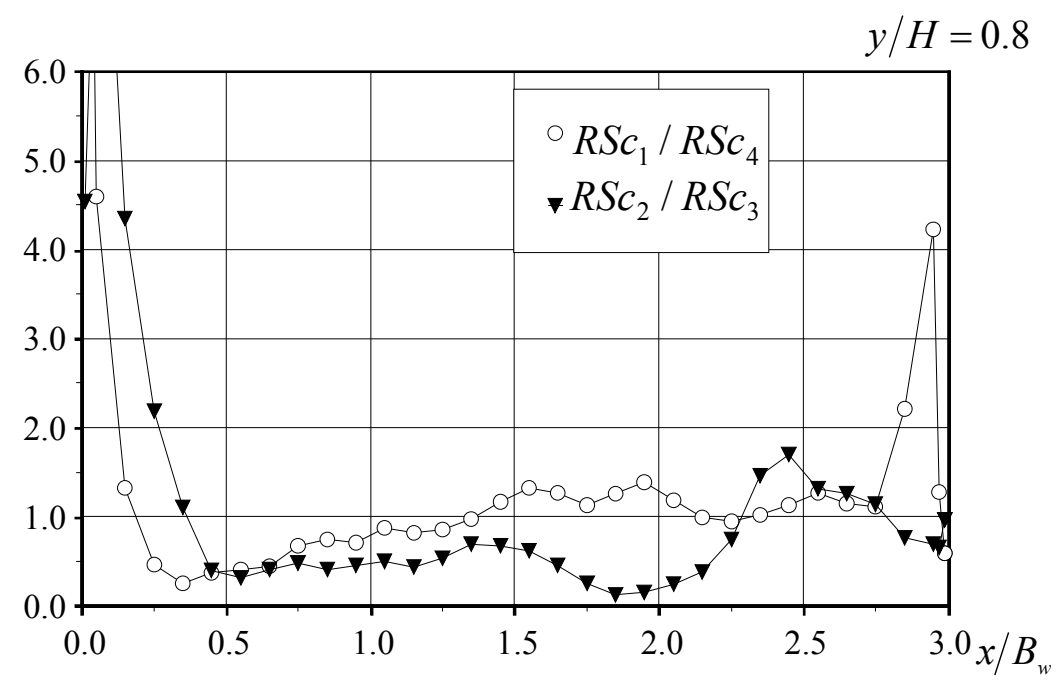

(e)

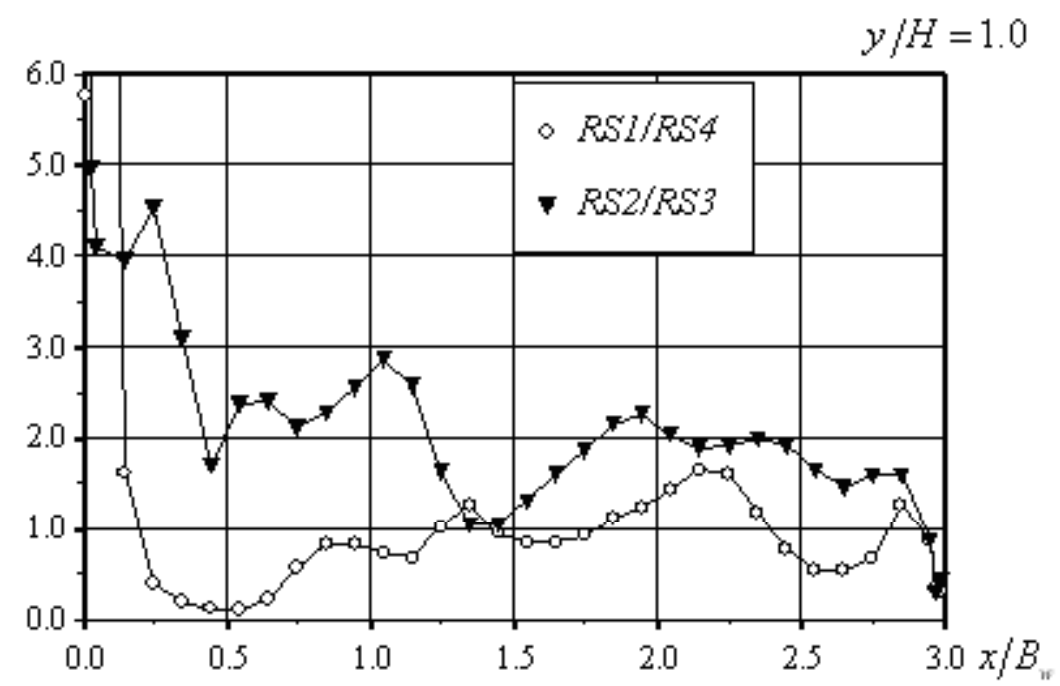

Fig.12 (a) to (d) Comparisons of quadrant Reynolds stress ratios 


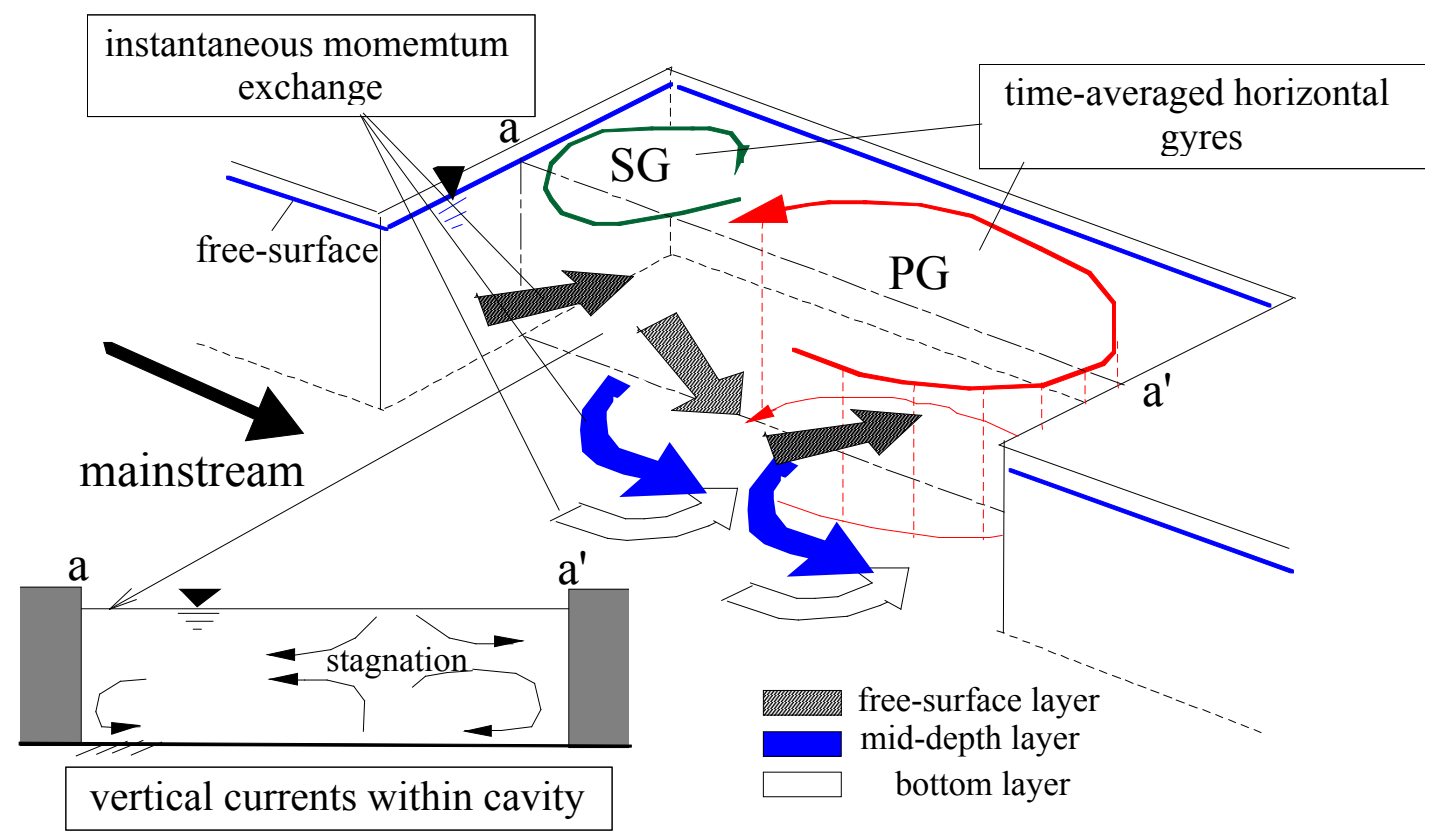

Fig.13 Phenomenological flow model in and around open-ended cavity zone 